EDITORIAL

\title{
Considerations about a thematic Supplement containing papers related to Translational Research in Surgery
}

\author{
Orlando de Castro e Silva Júnior
}

Full Professor, Department of Surgery and Anatomy, Ribeirão Preto Medical School, University of São Paulo, Ribeirão Preto, São Paulo, Brazil.

DOI: http://dx.doi.org/10.1590/S0102-86502016001300000

The present supplement was conceived and prepared starting in August 2015. There were papers already written which had not been submitted to other journals. Two had been submitted to and accepted on merit by two other other journals but at a cost exceeding 2,000.00 dollars each. Obviously we refused to pay this amount despite the high impact of the journals. Without controversy, it is our policy to pay for the publication of scientific papers only when we see that the amount is destined to cover the costs inherent to the editing of articles, and therefore not abusive, as collaboration with the journal involved.

At a meeting, Acta Cirúrgica Brasileira was the journal chosen for the submission of our papers that are part of this Supplement. Within six months we wrote the results obtained and we are now submitting them for possible publication. They are six papers in the coloproctology area, one in the area of digestive surgery, one in the area of urology and two in the cardiovascular area, all of them dealing with current topics of relevance in the area of surgery.

In general, we may consider this supplement to be thematic, dealing with cancer in different areas of surgery involving the introduction of new surgical techniques and studies on laser, methylene blue and hyperbaric oxygen therapy and their effects on the disease. Current sophisticated study methods were used in the various investigations, with relevant results and rich discussions, rendering the present supplement an excellent medium for consultation within the medical literature.

Regarding the use of laser, one of the papers specifically deals with the laser/hepatic tissue interaction in livers not submitted to any type of partial resection. The liver is a quiescent organ regarding cell proliferation: only 0.0012 to $0.01 \%$ of the hepatocytes are in mitosis at any given momemt ${ }^{2,3}$. The literature shows that toxic or surgical injuries in experimental models can alter this low cell turnover, leading to massive hepatocyte proliferation and consequently to the the total reestablishment of the hepatic mass within approximately two weeks after the loss of up to two thirds of the organ.

Several studies have shown an effective interaction of light with biological tissues, the liver in particular. Conventional light as well as LED (Light-Emitting Diode) and laser, with their different optical properties, have similar effects on the cell organelles, leading to increased efficiency of wound healing, of the hepatocellular mitochondrial energy cpacity and of hepatic regeneration. The tissue loss of part of the liver rapidly triggers the regenerative process until the originl weight is recovered. The observation of the response of the liver to various challenges such as hepatitis, intoxication, hepatic resection and liver transplantation is mythological and raises extremely interesting aspects regarding the hepatic coherence in overcoming challenges and obstacles. In particular, in this Supplement the authors studied the effect of laser light at two wavelengths on intact livers aiming at the possible application of a rational dose of laser light to the liver in various therapeutic modalities in the future.

We wish to thank Prof. Goldenberg for giving us the opportunity to publish this Supplement. 